\title{
Black Ascites: An interesting presentation of pancreatic duct leak
}

\author{
Mustafa S Ascha*1, Mona Ascha², Ibrahim A Hanouneh ${ }^{3}$ \\ ${ }^{1}$ Center for Clinical Investigation, Department of Epidemiology and Biostatistics, Case Western Reserve University, Cleveland, \\ Ohio, USA \\ ${ }^{2}$ Case Western Reserve University, School of Medicine, Cleveland, Ohio, USA \\ ${ }^{3}$ Minnesota Gastroenterology, Minneapolis, Minnesota, USA
}

Received: June 7, 2016

Accepted: July 31, 2016

Online Published: August 28, 2016

DOI: $10.5430 /$ crim.v3n $4 p 18$

URL: http://dx.doi.org/10.5430/crim.v3n4p18

\begin{abstract}
Introduction: Common causes of ascites include liver cirrhosis, heart failure, and malignancy. Pancreatic etiology is less common for ascites, but is most often a result of chronic pancreatitis secondary to alcoholic liver damage or pancreatic duct leak. Case description: The following case describes a patient with black-colored ascites secondary to pancreatic duct leak after total colectomy with ileorectal anastomosis. The colectomy was indicated by MYH-positive polyposis, and the there was no known prior liver or pancreas disease. Management included pancreatic duct stent placement, and drains from the pancreatic tail.

Discussion: Pancreatic etiology is rare among ascites diagnoses; this case is even rarer due to gross appearance of the ascitic fluid and its iatrogenic cause.
\end{abstract}

Key Words: Ascites, Pancreatic duct leak, Pancreatic ascites, Black ascites

\section{INTRODUCTION}

Ascites may be caused by cirrhosis (81\%), malignancy $(10 \%)$, and heart failure (3\%). ${ }^{[1]}$ For a patient with no history of liver disease presenting with ascites of unclear etiology, recommendations include a thorough history and physical exam, ultrasound, and diagnostic paracentesis. Imaging such as magnetic resonance imaging (MRI), computed tomography $(\mathrm{CT})$, endoscopic retrograde cholangiopancreatography (ERCP), and magnetic retrograde cholangiopancreatography (MRCP) may also be useful in diagnosis.

The abdominal exam may reveal masses concerning for malignancy, hepatomegaly or liver nodularity. Maneuvers such as a positive fluid wave and shifting dullness can confirm ascites, and a paracentesis can be performed for further diag- nostic testing. Ascitic fluid analysis includes gross inspection, total protein and albumin, cytology, cell counts, culture with gram staining, and fluid amylase. Most importantly, a serum-ascites albumin gradient (SAAG) greater than $1.1 \mathrm{can}$ be used as an indicator of portal hypertension.

Pancreatic ascites is an uncommon cause of abdominal ascites, accounting for approximately $1 \%$ of all cases. ${ }^{[1]}$ It was first reported in 1953 by Smith, who described two cases of chronic pancreatitis associated with ascites. ${ }^{[2]}$ In our case, the etiology is due to disruption of the main pancreatic duct, resulting in leakage and accumulation of pancreatic fluid in the peritoneal space. Pancreatic leaks or fistulas are classified as internal or external: external leaks are typically iatrogenic, while internal leaks may include pancreatic ascites, pleural

*Correspondence: Mustafa S Ascha; Email: mustafa.ascha@case.edu; Address: Center for Clinical Investigation, Case Western Reserve University, Cleveland, Ohio, USA. 
effusions, and pseudocysts. ${ }^{[3]}$

We present a unique case of pancreatic ascites after total abdominal colectomy with ileorectal anastomosis in a patient with no history of liver or pancreatic disease.

\section{Case presentation}

On day 10 after total abdominal colectomy with ileorectal anastomosis due to bi-allelic MYH-positive polyposis, a 60-year-old male presented with ascites that had caused significant discomfort. Of note, his colectomy had been complicated by polymorphous ventricular tachycardia and leukocytosis.

His past medical history was also significant for kidney impairment, elevated liver function tests (LFTs), benign neoplasms of the colon, portal vein thrombosis, and multiple thyroid nodules. He had no history of liver or pancreatic disease, was a never-smoker, and denied alcohol use.

Paracentesis yielded 5.5 liters of opaque dark brown (some clinicians claimed "black") ascitic fluid. An ascitic fluid analysis was negative for malignant cells, and demonstrated the following: 8,000 red blood cells (RBC), 1,296 nucleated cells, albumin $1.3 \mathrm{~g} / \mathrm{dl}$, amylase $1,178 \mathrm{U} / \mathrm{L}$, protein $2.7 \mathrm{~g} / \mathrm{dl}$. Serum amylase was $316 \mathrm{U} / \mathrm{L}$. Serum albumin was $3.2 \mathrm{~g} / \mathrm{dl}$, demonstrating a serum-ascites albumin gradient (SAAG) of 1.9. There were no organisms or growth on fluid culture and no polymorphonuclear leukocytes.

Abdominal CT imaging revealed subsegmental portal vein thrombosis in hepatic segment VIII, but otherwise no focal lesions and was unremarkable. MRCP detected a defect in the pancreatic tail with fluid signal extending anteriorly into adjacent peritoneum, suggesting a pancreatic duct leak. Thereafter, a pancreatic stent was placed and subsequent ERCP revealed no leak. On day 15 post-colectomy, CTguided drain placement $(\times 2)$ was performed at the tail of the pancreas with removal of $2.6 \mathrm{~L}$ of purulent fluid, for which cultures returned positive for Klebsiella pneumoniae.

On day 16 post-colectomy, the patient developed rigors and tachycardia, and subsequent laparoscopy was performed for abdominal cavity saline washout and placement of a left flank Blake drain. Three days later, the patient's vital signs were stable, however he demonstrated leukocytosis, diffuse abdominal pain, and diarrhea; the patient denied nausea, vomiting, fevers, chills, and rigors. After stent placement, ascites resolved.

\section{DISCUSSION}

Pancreatic ascites is frequently a result of chronic pancreatitis secondary to alcoholism, though $67 \%$ of patients present without recent acute pancreatitis exacerbation. ${ }^{[1]}$ Other more common causes include leaking pseudocyst, pancreatic duct fistula opening into the peritoneal cavity, or pancreatic duct rupture. There are reports of post-traumatic pancreatic ascites, in which the pancreatic tail was sheared off after trauma such as vehicle collisions or sports injury. ${ }^{[4]}$ However, patients typically have a history of chronic alcoholism, pancreatic disease, and liver disease. Pleural effusions may or may not be present, and pancreatic ascites is more common in males. ${ }^{[5]}$

\subsection{Diagnosis and treatment}

Pancreatic ascites is generally associated with ascitic fluid total protein greater than 3, amylase greater than 1,000 U/L, ascites/serum amylase ratio greater than 6 , and SAAG less than 1.1. Our patient met only the first criteria, with an amylase of 1,178 U/L, and pancreatic ascites was not confirmed until an MRCP revealed a pancreatic duct leak. Of note, our patient's ascitic fluid RBC count was significantly elevated at 8,000; the presence of this much blood in the ascites may make ascitic fluid parameters less reliable in the diagnosis. While not necessarily diagnostic, other characteristic findings of hemorrhagic ascitic fluid include an increased number of polymorphonuclear cells and erythrocytes, high specific gravity, and sterile bacterial cultures. In a report of 96 cases of pancreatic ascites, the majority $(n=74)$ of cases described the ascitic fluid as amber-colored. ${ }^{[5]}$

Other imaging that may be used to diagnose pancreatic ascites includes CT scan or ERCP to evaluate for pancreatitis or pseudocyst. ERCP is particularly beneficial as it is both diagnostic and therapeutic; after imaging, stent placement may occlude the disruption. Pancreatic ascites must be adequately treated and the underlying etiology resolved, lest pancreatic enzymes activate outside of the duodenum and digest peritoneal tissues and organs.

Medical therapy includes octreotide to reduce pancreatic secretions, while supportive therapy includes naso-gastric suctioning and total parenteral nutrition. However, these are temporary measures aimed at minimizing pancreatic secretions; permanent resolution is achieved with stent placement or surgical intervention. ${ }^{[3]}$

Given the patient's SAAG of 1.9, it could be argued that portal hypertension associated with portal vein thrombosis in hepatic segment VIII is the cause of his ascites. The gold standard test for portal hypertension is transjugular liver biopsy with porto-systemic pressure measurements. We did not believe this was indicated as we were not convinced the patient had portal hypertension, considering that there was no other evidence of liver cirrhosis or portal hypertension on 
imaging (such as collateral circulation of splenomegaly), the patient's AST to Platelet Ratio Index (APRI) formula was unremarkable, and the decreased accuracy of SAAG in the setting of hemorrhagic ascites caused by blood-ascitic fluid mixing.

\subsection{Black appearance}

To our knowledge, this is the first case of black pancreatic ascites following total abdominal colectomy with ileorectal anastomosis. A previous report describes similar blackappearing peritoneal dialysate in a patient with end-stage renal disease. ${ }^{[6]}$ Black peritoneal dialysate has also previously been described in patients with fungal peritonitis due to Curvularia species. ${ }^{[7]}$

The differential diagnosis for black ascites can include bowel perforation with leakage of fecal matter, fungal peritonitis, metastatic melanoma, primary ovarian carcinoma, leakage of tattoo ink. ${ }^{[6-8]}$ Leakage of fecal matter was a possibility in our patient, however no evidence of perforation, such as pneumoperitoneum, was found on imaging. The patient had no history of melanoma, and primary ovarian carcinoma was not possible due to the male sex of the patient. It has been suggested that India ink may leak into the peritoneum and cause black appearing effluent, but no tattooing procedure was done in this patient. ${ }^{[6]}$

Non-pancreatic diagnoses have been noted to cause opaque, dark brown ascites. One such diagnoses is endometriosis, which was ruled out due to the male sex of this patient. ${ }^{[9]}$ Another case report describes brown-black ascites in a patient with eosinophilic ascites, though eosinophilic ascites was ruled out in this patient due to normal peripheral eosinophil counts and normal ascites polymorphonuclear leukocyte counts. ${ }^{[10]}$

We hypothesize that the high RBC count in the ascitic fluid caused the effluent to appear black, particularly in the environment of proteolytic enzymes released from the pancreatic duct leak. Such enzymes provide a suitable milieu for the production of methemalbumin, which can appear brownish to black and has been found in ascitic fluid of at least one patient with acute hemorrhagic pancreatitis. ${ }^{[11]}$

\section{Conclusions}

Pancreatic ascites is not commonly seen in patients with no prior history of liver disease, pancreatic disease, or history of alcoholism. There have been reports of blunt abdominal trauma which led to pancreatic pseudocyst formation and pancreatic ascites, and even fewer reports of iatrogenic pancreatic ascites. ${ }^{[5]}$ It has been reported that traumatic pancreatic ascites has a faster onset (several weeks to several months) than non-traumatic pancreatic ascites (several weeks to one year). ${ }^{[5]}$ Iatrogenic pancreatic ascites is a rarely reported event. A literature search revealed one case report describing a patient who underwent endoscopic ultrasonography-guided fine needle aspiration for evaluation of a pancreatic neck mass with subsequent development of a pancreatic duct leak causing pancreatic ascites. ${ }^{[12]}$

Furthermore, the patient did not meet the clinical criteria of pancreatic ascites, but this is thought to be due to the fact that the high RBC count in the ascitic fluid caused the ascitic fluid parameters to be less reliable. The SAAG measure, also, may be less reliable for patients with hemorrhagic ascites. Thus, diagnostic value of paracentesis was reduced, and we recommend a low threshold for confirmation of a pancreatic duct leak with ERCP or MRCP in patients with high ascitic fluid RBC count.

\section{Conflicts of InTEREST Disclosure}

The authors have declared no conflicts of interest.

\section{REFERENCES}

[1] Montemuro P, Roy A. Not Your Typical Case Of Ascites: Pancreatic Ascites In A Patient With Cirrhosis And Pancreatic Duct Leak. The Medicine Forum. 2012; 13.

[2] Smith EB. Hemorrhagic ascites and hemothorax associated with benign pancreatic disease. Arch Surg. 1953; 67: 52-56. http: //dx.doi.org/10.1001/archsurg. 1953.01260040055008

[3] Larsen M, Kozarek R. Management of pancreatic ductal leaks and fistulae. J Gastroenterol Hepatol. 2014; 29(7): 1360-70. http: //dx.doi.org/10.1111/jgh. 12574

[4] Kozarek RA. Management of pancreatic ascites. Gastroenterol Hepatol (NY). 2007; 3(5): 362-4.

[5] Hotz J, Goebell H, Herfarth C, et al. Massive pancreatic ascites without carcinoma. Report of three cases. Digestion. 1977; 15(3): 200-16. http://dx.doi.org/10.1159/000198004

[6] Headley CM, Naseer A, Ramaiah M, et al. "Black"-Appearing Peritoneal Effluent. Nephrol Nurs J. 2015; 42(6): 569-75. PMid:26875232

[7] Charlton JR, Barcia JP, Norwood VF. Black Specks in Dialysis Fluid: An Unusual Case of Peritonitis in a Pediatric Patient on Peritoneal Dialysis. Dial. Transplant. 2010; 39: 445-448. http: //dx.doi.org/10.1002/dat. 20491

[8] Yeh KE, Marcus PS, Fong TL. Paraneoplastic Cholestasis Associated With Ovarian Dysgerminoma. Obstet Gynecol. 2015; 126(2): 431-4. PMid:25923022 http://dx.doi.org/10.1097/A0G.000 0000000000699

[9] Bhojawala J, Heller DS, Cracchiolo B, et al. Endometriosis presenting as bloody pleural effusion and ascites-report of a case and 
review of the literature. Arch Gynecol Obstet. 2000; 264(1): 39-41. PMid:10985620 http://dx.doi.org/10.1007/PL00007484

[10] Chandrasekar TS, Goenka MK, Lawrence R, et al. An unusual case of ascites. Indian J Gastroenterol. 2012; 31(4): 203-7. PMid:23001826 http://dx.doi.org/10.1007/s12664-012-0204-2

[11] Geokas MC, Rinderknecht H, Walberg CB, et al. Methemalbumin in the diagnosis of acute hemorrhagic pancreatitis. Ann Intern Med. 1974; 81(4): 483-6. PMid:4414654 http://dx.doi.org/10.73 26/0003-4819-81-4-483

[12] Reddymasu S, Oropeza-vail MM, Williamson S, et al. Pancreatic leak after endoscopic ultraound guided fine needle aspiration managed by transpapillary pancreatic duct stenting. JOP. 2011; 12(5): 489-90. PMid:21904078 